\title{
Endoscopic Hemostasis of a Pleural Hemorrhage using a Hemostatic Gel
}

\author{
Lucian Negreanu ${ }^{1}$, Florin Chirculescu ${ }^{2}$, Loredana Goran ${ }^{1}$
}

1) Department of Gastroenterology, University Hospital, 'Carol Davila’ University Bucharest; 2) Department of Thoracic Surgery, University Hospital, Bucharest, Romania

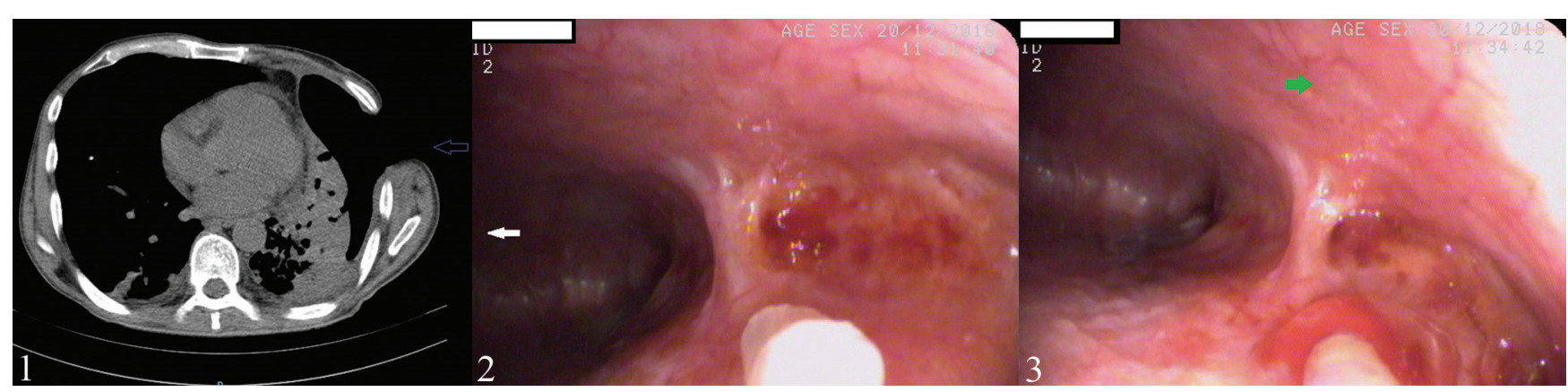

A 54-year-old man presented with bleeding from the pleural cavity after a blind pleural biopsy. He was previously diagnosed with fibrous-cavernous pulmonary tuberculosis and left pleural empyema and had received an Eloesser flap thoracostomy window procedure due to difficulties in resolving pulmonary lesions. An open thoracic window (blue arrow on CT scan, Fig. 1) facilitated the resolution of the infected pleural collection [1].

With the patient in a supine position, we introduced a Pentax EG290 upper endoscope through the thoracic window into the pleural cavity and noticed oozing bleeding near an adhesion on the thoracic pleural side (Fig, 2, white arrowvisceral pleura). Using the scope, we applied PuraStat ${ }^{\oplus}$ gel over the bleeding area via a catheter, successfully achieving hemostasis. The advantage of PuraStat ${ }^{\oplus}$ is that it is perfectly transparent and the lesions remain visible all the time during and after its application (Fig 3, thoracic pleura, green arrow). The patient was discharged the next day post-procedure without bleeding recurrence. We chose PuraStat ${ }^{\oplus}$ instead of the more widely used Hemospray in order to avoid introduction of a hemostatic powder into the pleural space.

PuraStat $^{\oplus}$, an extracellular scaffold matrix, consists of four amino acids which convert into a polypeptide chain that assembles into fibers upon contact with blood or body fluids, resulting in a translucent hydrogel. The hemostatic role of the synthetic self-assembling peptide is accomplished by physically closing the ruptured blood vessels. PuraStat ${ }^{\oplus}$ was recently introduced in gastroenterology for use after endoscopic mucosal resections or endoscopic submucosal dissections [2, 3], proving its efficacy in patients with a high risk of bleeding (antiplatelet/ anticoagulant therapies, portal hypertension) [2]. PuraStat ${ }^{\circledR}$ efficiency was also confirmed in a dual center study, in which conventional endoscopic techniques failed to stop the bleeding in six patients with massive lower gastrointestinal bleeding [4].

This is the first case of using PuraStat ${ }^{\oplus}$ in the pleural cavity to control bleeding in a patient with an open thoracic window for pleural empyema. It proved to be safe, easy to use and effective in controlling bleeding.

Corresponding author: Lucian Negreanu,negreanu_99@yahoo.com

Conflicts of interest: None to declare.

\section{REFERENCES}

1. Carrillo EH, Barkoe DJ, Sanchez R, et al. Open thoracic window: A useful alternative for retained infected pleural collections in critically ill trauma patients. Am Surg 2009;75:152-156.

2. Pioche $M$, Camus $M$, Rivory J, et al. A self-assembling matrix-forming gel can be easily and safely applied to prevent delayed bleeding after endoscopic resections. Endosc Int Open 2016;4:E415-E419. doi:10.1055/s-0042-102879

3. Subramaniam S, Kandiah K, Thayalasekaran S, Longcroft-Wheaton G, Bhandari P. PTH-019 Bleeding during endoscopic resection: a novel extracellular scaffold matrix is a safe and effective haemostatic agent. Gut 2017;66:A214-A215.

4. De Nucci G, Omazzi B, Domenico Mandelli E, et al. Purastat, a new hemostatic device to control massive GI bleedings: a dual centre case series. Endoscopy 2018;50:S175-S176. doi:10.1055/s-0038-1637571 